\title{
The Effectiveness of Cognitive Rehabilitation on Anxiety Reduction and Brainwave Pattern in Patients with Anxiety Disorder
}

\author{
Raheb Jafari ${ }^{1, *}$, Hassan Bafandeh ${ }^{2}$ \\ ${ }^{1}$ Master of Science in Cognitive Science, Shahid Madani University of Azarbaijan, Tabriz, Iran \\ ${ }^{2}$ Assistant Professor, Shahid Madani University of Azarbaijan, Tabriz, Iran \\ * Corresponding author: Raheb Jafari, Master of Science in Cognitive Science, Shahid Madani University of Azarbaijan, Tabriz, \\ Iran. E-mail: Raheb.jafari2019@yahoo.com \\ How to Cite this Article: \\ Jafari R, Bafandeh H. The Effectiveness of Cognitive Rehabilitation on Anxiety Reduction and Brainwave Pattern in Patients with \\ Anxiety Disorder. Iran J Rehabil Res Nurs. 2020;7(1):66-74. \\ DOI: 10.29252/ijrn.7.1.66
}

Received: 09 Aug 2019 Accepted: 16 Apr 2020 Keywords:

Cognitive Rehabilitation Anxiety

Brain Waves

(C) 2020 Iranian Journal of

Rehabilitation Research in Nursing

\begin{abstract}
Introduction: Studies have shown that brain waves are anomalously active in anxiety patients, but so far, no interventional studies have been performed. Therefore, the purpose of the present study was to determine the effectiveness of cognitive rehabilitation on reducing anxiety and brain wave pattern in patients with anxiety. Anxiety disorder was widespread.

Methods: This study was quasi-experimental. The statistical population of this study was that all patients with generalized anxiety disorder referred to Tabriz psychiatric centers in 2018. Thirty anxious patients were selected after the psychiatrist's diagnosis and were randomly assigned to two experimental and control groups. Then both groups were assessed for alpha and theta wave PZ area as well as anxiety severity (Beck Anxiety Inventory) (pre-test). Then the experimental group underwent cognitive rehabilitation for 12 sessions of 60 minutes. They received the relevant training, and in the end, in order to assess the differences, the above tests were repeated on both experimental and control groups (post-test). Data were analyzed using Neuroguid and SPSS 23 software.

Results: The analysis of the present data showed that cognitive rehabilitation reduced anxiety scores in patients with a generalized anxiety disorder $(\mathrm{P}<0 / 01)$ and also reduced the alpha wave and theta wave of anxiety in $P Z$ patients $(P<0 / 01)$.

Conclusions: Based on results, cognitive rehabilitation can be used as an effective treatment for reducing anxiety.
\end{abstract}

\section{Extended Abstract}

\section{OBJECTIVE}

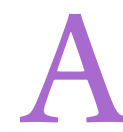
nxiety is one of the most common diseases caused by symptoms such as palpitations, tremors, tremors, and activation of the sympathetic branch [1]. Among psychological disorders, anxiety disorders are common [2]. One type of anxiety disorder is pervasive anxiety disorder [1]. Which is a common and often chronic disorder of psychiatry [3]. In the Diagnostic and Statistical Manual of Mental Disorders (Fifth Edition), pervasive anxiety disorder includes anxiety, worry, and extreme stress that is seen on most days, lasts for at least six months, and is the focus of a person's everyday life events. This anxiety is difficult to control. It causes inner suffering for the person and disrupts essential areas of the person's life [4]. In recent decades, there has been a growing interest in using rehabilitation programs in the field of cognitive problems, which has led to the expansion of cognitive training programs so that these programs can adjust the level of difficulty of the task. Simple problems are based on individual differences and create ongoing cognitive challenges for patients [15]. Cognitive rehabilitation refers to training that seeks to perform cognitive functions based on cognitive science findings (accuracy, attention, visual-spatial perception, auditory cleanliness, 
memory types, especially working memory, etc.). Improve or enhance other executive functions, all of which point to the principle of neural and cerebral flexibility [16].

\section{MATERIALS AND METHODS}

This study was a quasi-experimental study with a pretest-post-test and control group. After receiving the necessary letters of introduction, the code of ethics was applied, and this research was done with the code of ethics of IR.IAU.tabriz.1398.10. The statistical population of the present study included all male patients with symptoms of a generalized anxiety disorder who referred to psychiatric centers in Tabriz in 2018 in the age group of 25 to 30 years and from the beginning of July to the end of January. The sample of the present study included 30 anxious patients who were purposefully selected from the mentioned community. After being diagnosed with a pervasive anxiety disorder by a psychiatrist, the patients were first tested for anxiety and recorded a small amount of electroencephalography in the $\mathrm{PZ}$ region at alpha and theta frequencies. The experimental group then underwent cognitive rehabilitation for 12 sessions, and no intervention was performed for the control group. Rehabilitation sessions lasted three months a week. It was done after the posttest sessions. Because gender is influential in the field of pervasive anxiety, only men were used to control it.

\section{RESULTS}

Multivariate analysis of covariance was used to analyze the data of this study. This test is used to control the effect of the pre-test.

Table 1. The Results of Multivariate Analysis of Covariance for Each Variable in the Text of Multivariate Analysis of Covariance with the Aim of Determining the Differences between the Two Groups in Anxiety, PZ Alpha and Theta PZ

\begin{tabular}{lccccc}
\hline Variable & CS & MS & F & Pta \\
\hline Anxity & $937 / 56$ & $937 / 56$ & $201 / 74$ & $0 / 001$ & $0 / 27$ \\
Alphapz & $241 / 10$ & $241 / 10$ & $177 / 13$ & $0 / 001$ & $0 / 12$ \\
Thetapz & $324 / 14$ & $324 / 14$ & $317 / 57$ & $0 / 001$ & $0 / 19$ \\
\hline
\end{tabular}

As can be seen in Table 1 , cognitive rehabilitation reduces anxiety, alpha, and theta $\mathrm{PZ}$, and is significantly different at the $\mathrm{P}<0.001$ level.

\section{CONCLUSION}

Explaining the effectiveness of cognitive rehabilitation on reducing anxiety, it can be said that cognitive rehabilitation program significantly increases processing speed, cognitive flexibility, and memory, and plays a significant role in reducing the activity of the parietal cortex at alpha and tetra frequencies. It is also one of the types of brain training that uses a particular program that aims to increase cognitive skills or create cognitive ability by performing exercises that can cause significant changes in behavioral levels, attention, memory and other executive actions become the brain. Given the close relationship between executive action and anxiety disorders, the cognitive rehabilitation program can be effective in improving anxiety symptoms [21]. Explaining the effectiveness of rehabilitation on reducing alpha and theta activity in the $\mathrm{PZ}$ region, it can be said that anxious patients have a malfunction of the parietal lobe, and this region is more active than the brain. So it is not uncommon for these people to have low-level cognitive functions, which in turn can exacerbate anxiety. As a result, cognitive rehabilitation training by improving brain function, in addition to improving attention, concentration, and information processing, also reduces alpha and PZ activity [24].

\section{Ethical Considerations}

\section{Compliance with ethical guidelines}

This study was a quasi-experimental study with a pretest-post-test and control group. After receiving the necessary letters of introduction, the code of ethics was applied, and this research was done with the code of ethics of IR.IAU.tabriz.1398.10. All subjects were given ethical consent and assured that the results would remain confidential and that the results would only be confidential A group will be analyzed.

\section{Funding}

Cognitive rehabilitation, with the continuous arousal of less active areas in the brain of people with anxiety, changes the synaptic stability based on the principle of formability and self-healing of the brain. Based on the principle of brain formability, the reason for the persistence of changes caused by cognitive rehabilitation can be attributed to the structural or functional changes created in people's brains through cognitive training.

\section{Authors' contributions}

Mr. Raheb Jafari was in charge of collecting data and writing the article, and Mr. Hassan Bafandeh was in charge of analyzing the statistical information.

\section{Conflicts of Interest}

The authors of this article are all members of the Shahid Madani University of Azerbaijan, so in order to avoid conflict of interest, do not send the article for judging to the judges of the faculty member of the mentioned university. The authors did not receive any financial support from any institution, organization, or body to conduct this research.

\section{Acknowledgments}

The authors consider it necessary to thank the researchers of Tabriz Psychiatric Centers and Valiasr Counseling Center of Tabriz, who provided the possibility of holding medical sessions to all researchers and all participants in the present study. 


\title{
اثربخشى توانبخشى شناختى بر كاهش علايم اضطراب فراكير و الكوى امواج مغزى در بيماران مبتلا به اختلال اضطراب
}

\author{
راهب جعفرى ا“**، حسن بافنده r
}

' كارشناس ارشد علوم شناختى، دانشكاه شهيد مدنى آذربايجان، تبريز، ايران

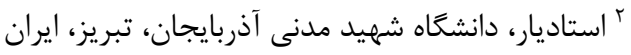

* نويسنده مسئول: راهب جعفرى، كارشناس ارشد علوم شناختى، دانشخاه شهيد مدنى آذربايجان، تبريز، ايران. ايميل: Raheb.jafari2019@yahoo.com

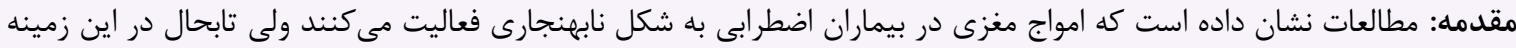

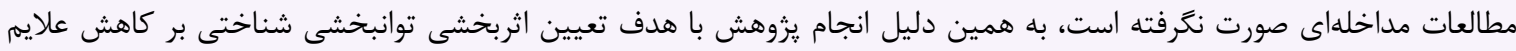

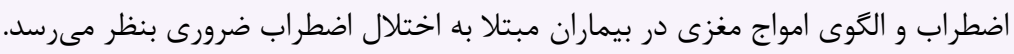

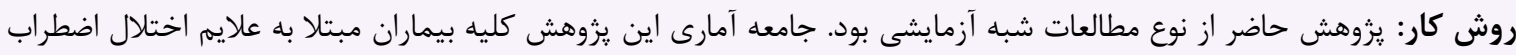

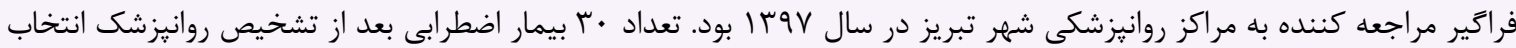

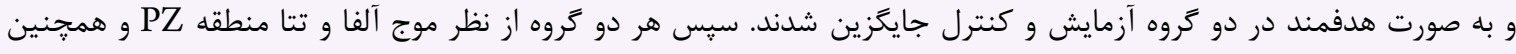

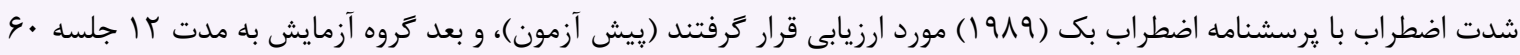

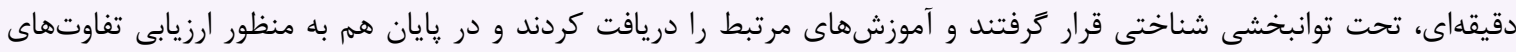

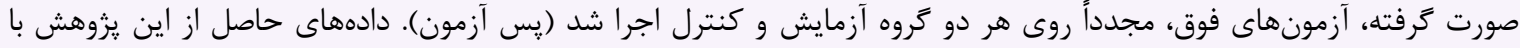

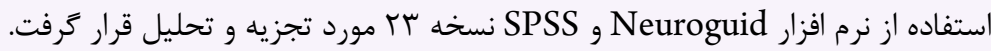

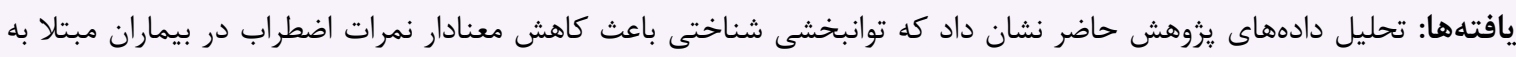

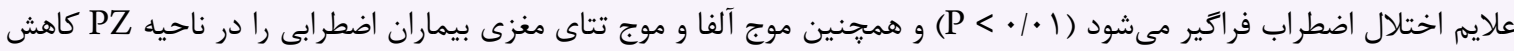

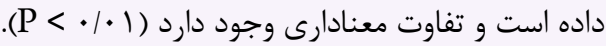

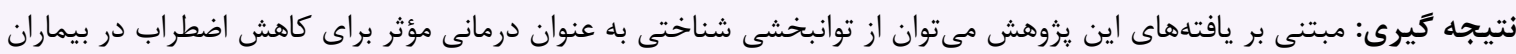

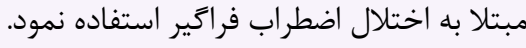

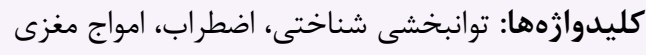

تمامى حقوق نشر براى انجمن علمى يرستارى ايران محفوظ است.

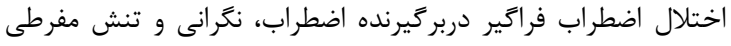

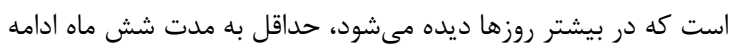

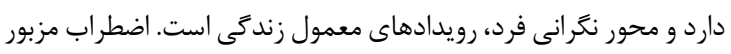

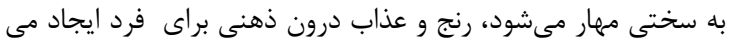

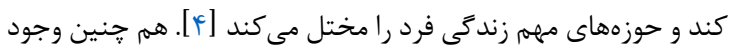

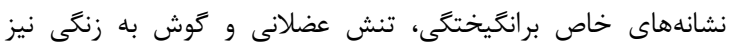

اضطراب، يكى از بيمارىهاى شايع محسوب مىشود كه با نشانكانى

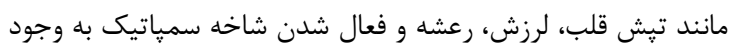

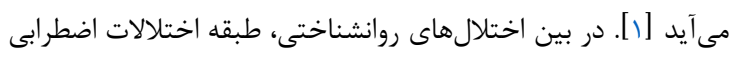

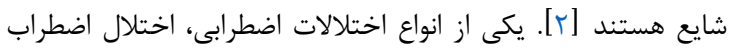

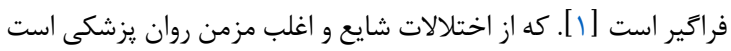

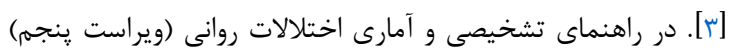


در يروهشى شيرازى و همكاران نتايج نشان داد كه آموزش توانبخشى

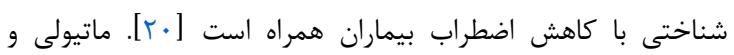

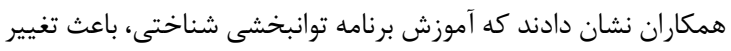

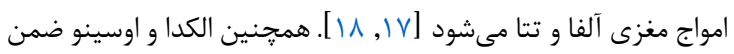

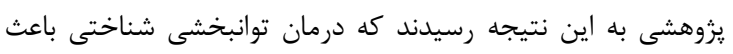

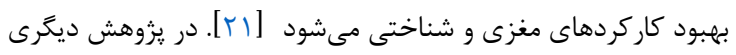

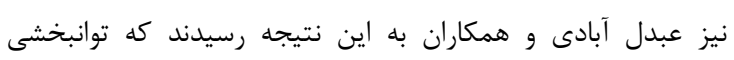

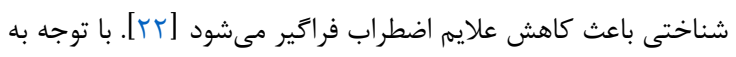

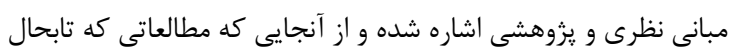

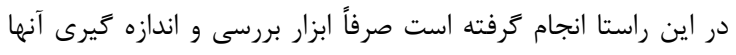

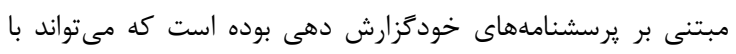

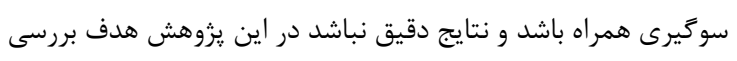

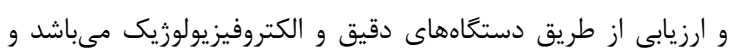

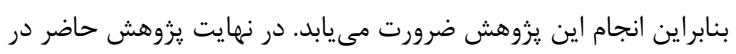

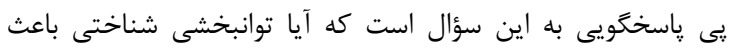

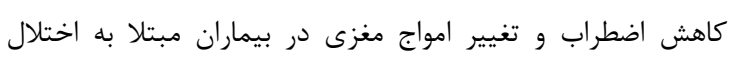

اضطراب فراخير مىشود؟ اضطراب تونير

\section{روش كار}

اين يروهش از نوع مطالعات شبه آزمايشى با ٍِيش آزمون- يس آزمون

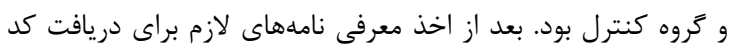

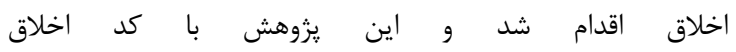
IR.IAU.tabriz.1398.10 انجام شد. جامعهى آمارى ئروهش

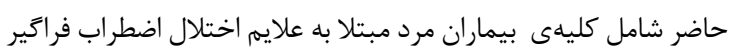

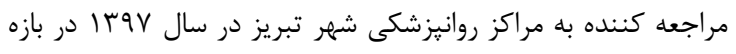

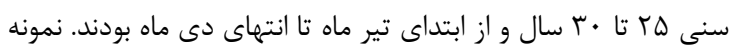

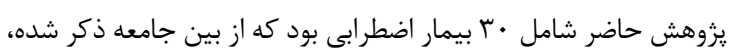

$$
n=\frac{N z^{2} p q}{N d^{2}+z^{2} p q}
$$

بعد از تشخيص اختلال اضطراب فراگير توسط روان يزشك، ابتدا از اين

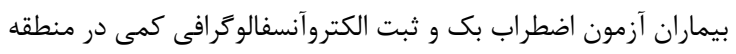

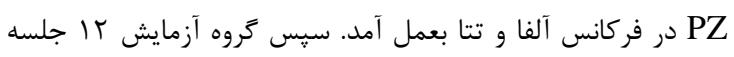

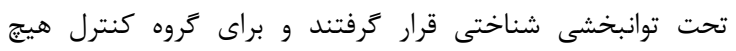

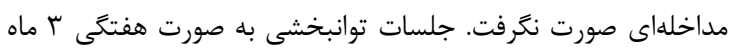

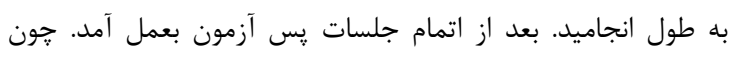

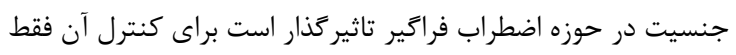

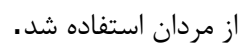

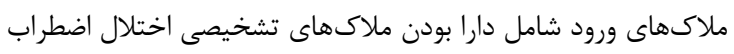

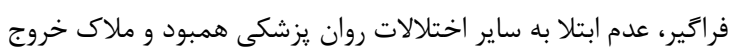

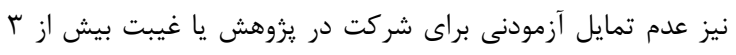

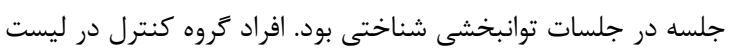

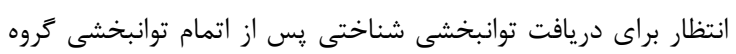
آزمايش قرار كرفتند.
ضرورى ذكر شده است و اضطراب و نكَرانى بايد با سه يا بيش از سه

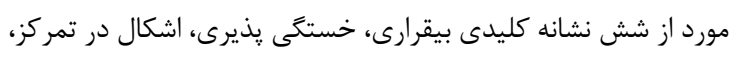

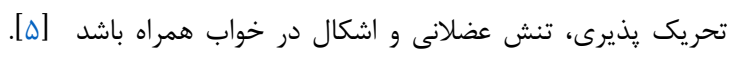

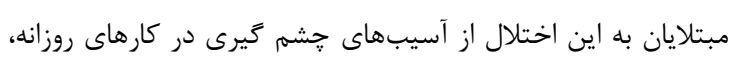

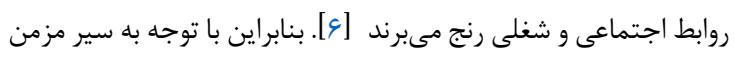

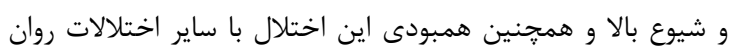

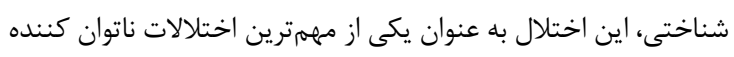

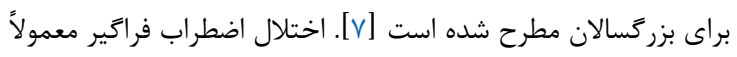

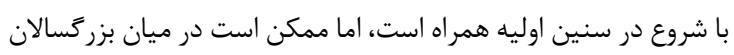

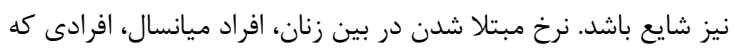

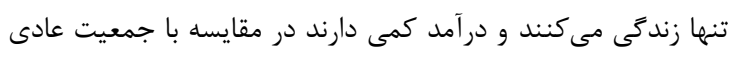

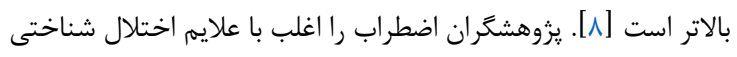

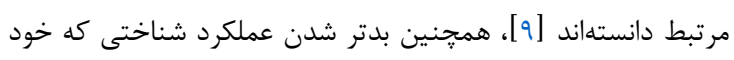

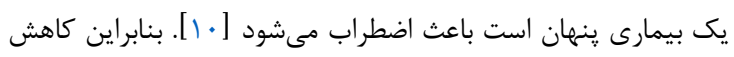

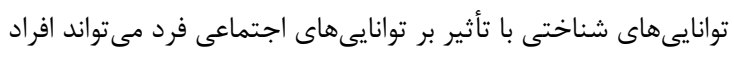
راكر معرض خطر اضطراب قرار دهد [11]

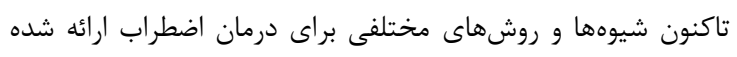

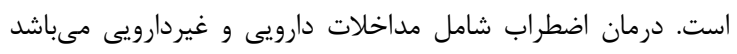

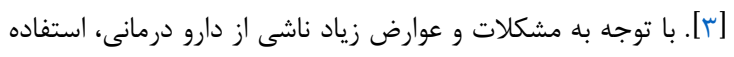

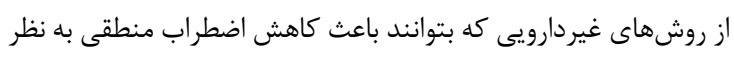

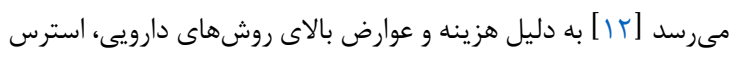

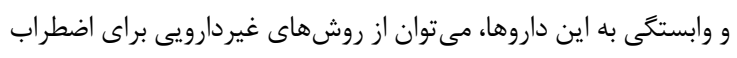

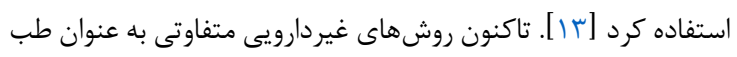

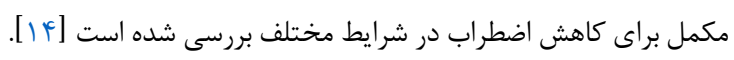

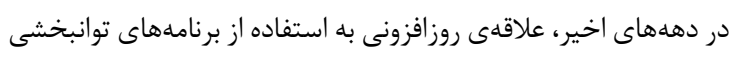

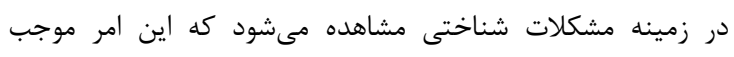

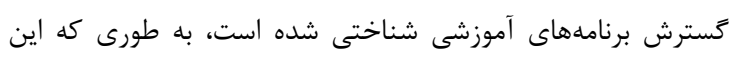

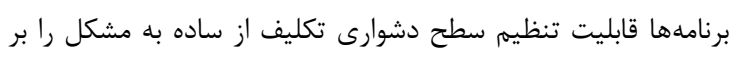

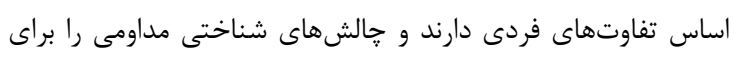

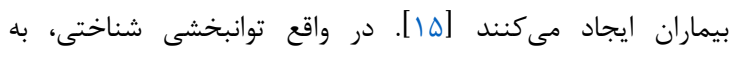

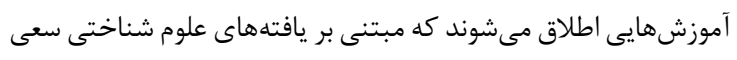

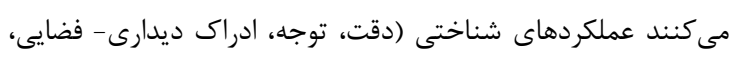

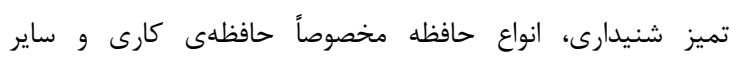

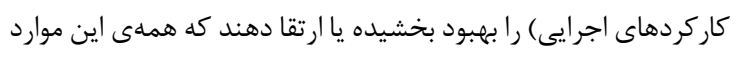

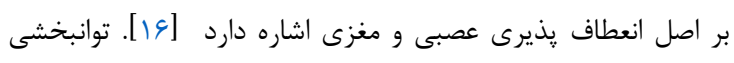

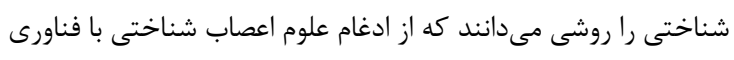

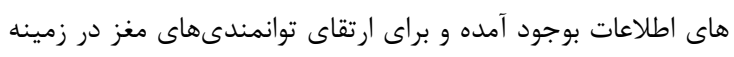
ى كاركردهاى شناختى از جمله ادراك، توجه، هوشيارى، حافظه و ...

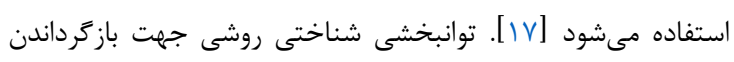
ظرفيتهاى شناختى از دست رفته است كه توسط تمرينات و ارائه

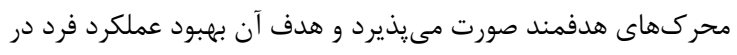

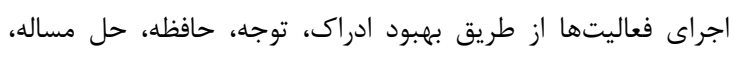

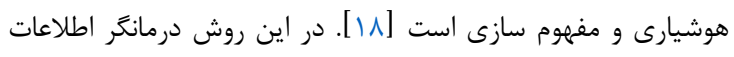

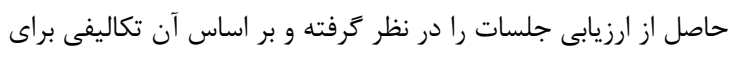

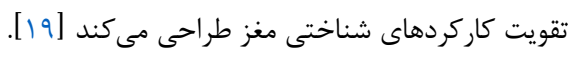


[rr]]. يرسشنامه آT ماده دارد كه به صورت صفر (نشانه سلامت روان)

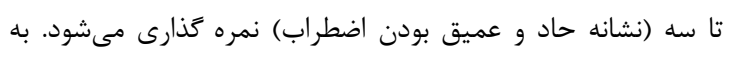

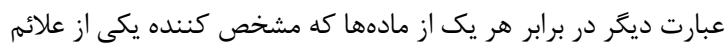

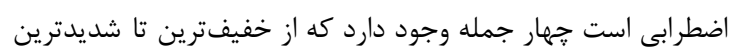

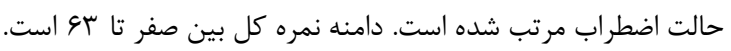

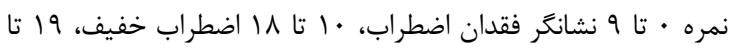

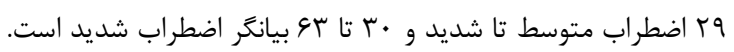

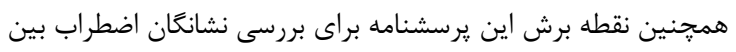

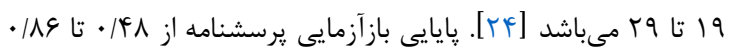

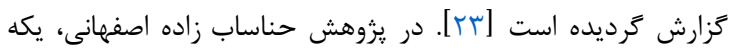

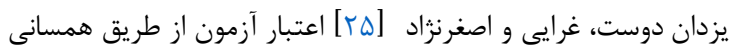

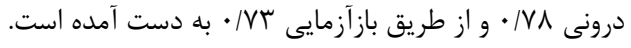

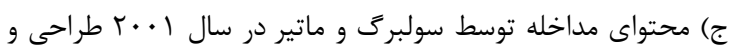

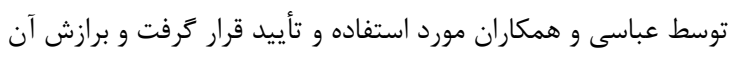

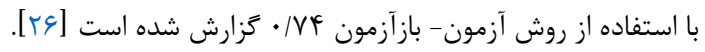

ابزار اندازه كيرى شامل: الف) الكتروآنسفالوًرافى كمى: براى ثبت موج

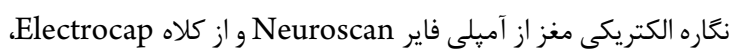

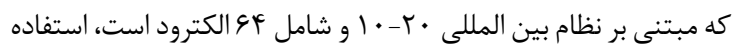

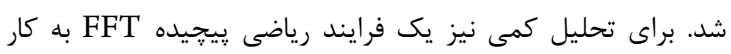

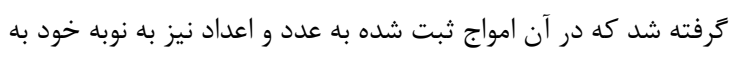

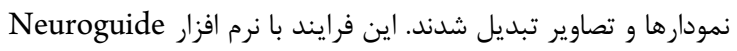

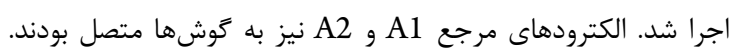

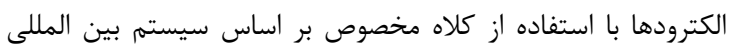

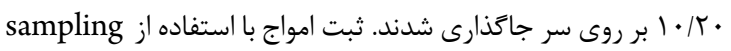

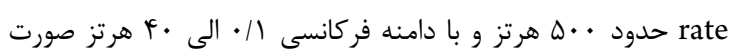

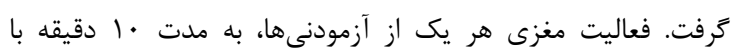

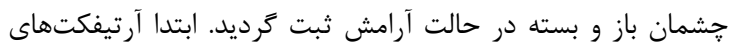

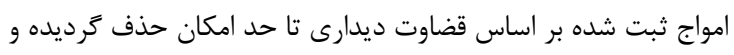

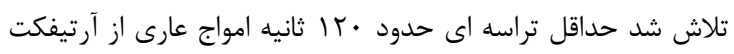
جهت تحليل در اختيار باشد.

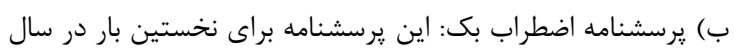

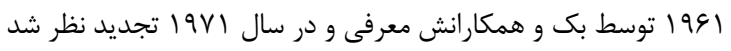

\begin{tabular}{|c|c|}
\hline \multicolumn{2}{|c|}{ جدول ا. شرح جلسات توانبخشى به تفكيك هر جلسه } \\
\hline شرح جلسات & جلسات \\
\hline آشنايى و برقرارى اتحاد درمانى، بيماران با روشها و تمرينهاى شناختى آشنا و خط باسه كاركردهاى شناختى آنان اندازه كيرى شد. & جلسه اول \\
\hline به تمرينهاى توجهى شامل كوش به زنكى با محر كهاى شنيدارى (اعداد، كلمات، جملات و غيره) و بررسى و تهيه رويدادهاى استرس زاى زندكى اختصاص يافت. & جلسه دوم \\
\hline كاربركى مرتبط با توضيحات توانبخشى ارائه و افزايش مهارت عناصر توجهى و تمرينهاى توجهى شامل حفظ توجه آموزش داده شد. & جلسه سوم \\
\hline به تمرينهاى توجهى ديدارى و شنيدارى و تمرينهاى حافظه اختصاص يافت. & جلسه جهارم \\
\hline تمرينهاى مربوط به تغيير توجه، درك مطلب و بسط شناختى آموزش داده شد. & جلسه ينجم \\
\hline تمرينهاى رعايت نظه نزولى و صعودى جهت حفظ توجه و خواندن براى درى مطلب و جستجوى همزمان حروف و كلمات از قبل تعيين شده انجام شد. & جلسه ششم \\
\hline 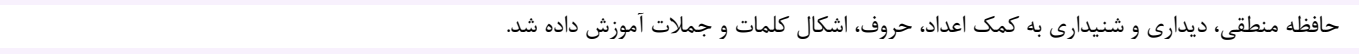 & جلسه هفتم \\
\hline به بهببود حافظه كلامى و تصويرى، سازماندهى كلامى و ساخت تداعىهاى زوجى اختصاص داده شد. & جلسه هشتم \\
\hline تمرينهاى عملكرد اجراى شامل بيان طرح مربوط به يك تكليف ساده، طبقه بندى، تمايز قائل شدن، حل مساله و خود دستوردهى آموزش داده شد. & جلسه نهم \\
\hline 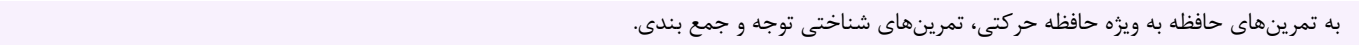 & جلسه دهم \\
\hline 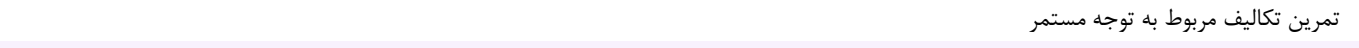 & جلسه يازدهم \\
\hline تمرين تكاليف مربوط به توجه متمركز و يراكنده & 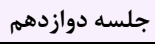 \\
\hline
\end{tabular}

ولى در مرحله يس آزمون گروههاى آزمايشى، اين كميتها با واريانس

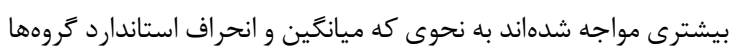
تغيير ييدا كرده است.

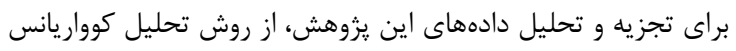

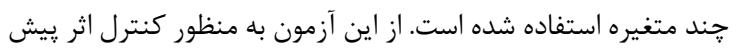

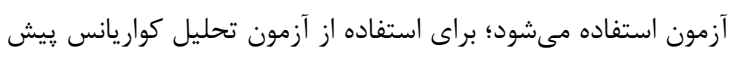

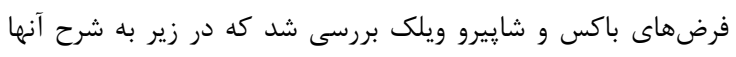

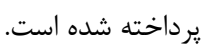
از آزمون باكس براى بررسى همَّنى ماتريس كوواريانس استفاده شد؛

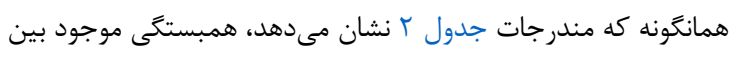

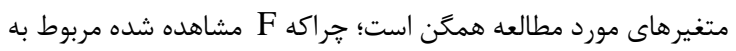

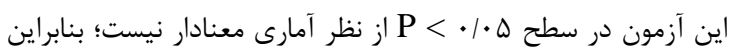
بيش فرض همخنى ماتريس كوراريانس محقق شده است.
دادههاى بدست آمده از يزوهش حاضر با استفاده از روشهاى آمار

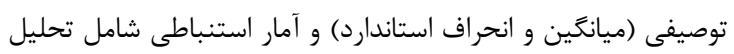

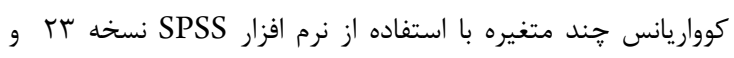
همجنين نرم افزار Neuroguid تجزيه و تحليل شدند.

يافتهنا

ميانكين و انحر اف معيار سن شركت كنندكان در گروه مداخله برابر با

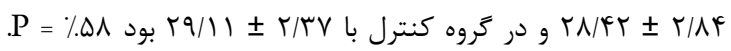

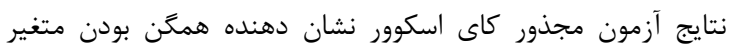

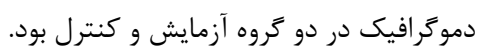

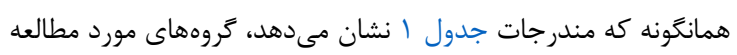

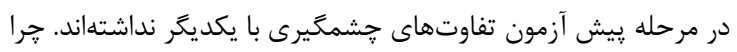

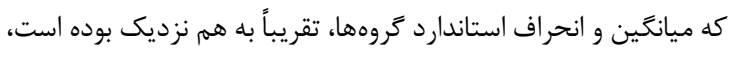




\begin{tabular}{|c|c|c|c|c|c|c|}
\hline \multicolumn{3}{|c|}{ يَس_آزمون } & \multicolumn{3}{|c|}{ يِيش آزمون } & \multirow[t]{2}{*}{ آزمودنىها } \\
\hline تعداد & انحراف استاندارد & ميانكين & ت تعداد & انحراف استاندارد & ميانكين & \\
\hline & & & & & & ضطراب \\
\hline 10 & $4 / 9 V$ & TQ/QT & 10 & $F / A \varphi$ & HT/AS & آزمايش \\
\hline \multirow[t]{2}{*}{10} & $4 / \cdot 1$ & Tr/gT & 10 & $F / v r$ & rT/lT & كنترل \\
\hline & & & & & & كZ \\
\hline 10 & r/gr & $1 / 48$ & 10 & $r / \Delta \varphi$ & $11 / 1$. & آزمايش \\
\hline \multirow[t]{2}{*}{10} & $r / T F$ & $11 / \Delta r$ & 10 & $r / \Delta 9$ & $11 / \mathrm{rr}$ & كنترل \\
\hline & & & & & & تتا PZ \\
\hline 10 & $\cdot / \Lambda r$ & F/QT & 10 & $1 / 19$ & S/VT & آزمايش \\
\hline 10 & $r / . r$ & $V / .9$ & 10 & $1 / 29$ & s/9r & كنترل \\
\hline
\end{tabular}

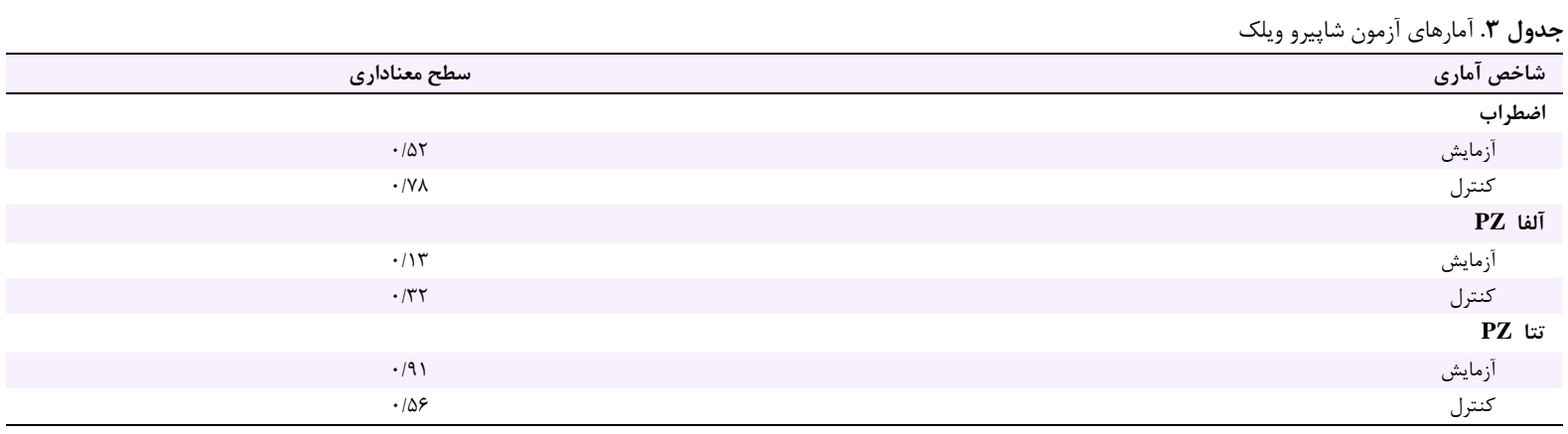

\begin{tabular}{|c|c|c|c|c|c|}
\hline & & & رر متغيرهاى وابسته & ل فمخنح & حدول F. آزمون ام \\
\hline $\mathbf{P}$ & $\mathbf{F}$ & درجه آزادى دوم & درجه آزادى اول & ام باكس & متغير \\
\hline.$/ 410$ & $1 / \cdot 1$ & $\Delta \& \wedge \cdot / \Gamma$ & 4 & $9 / 11$ & متغير هاى وابسته \\
\hline
\end{tabular}

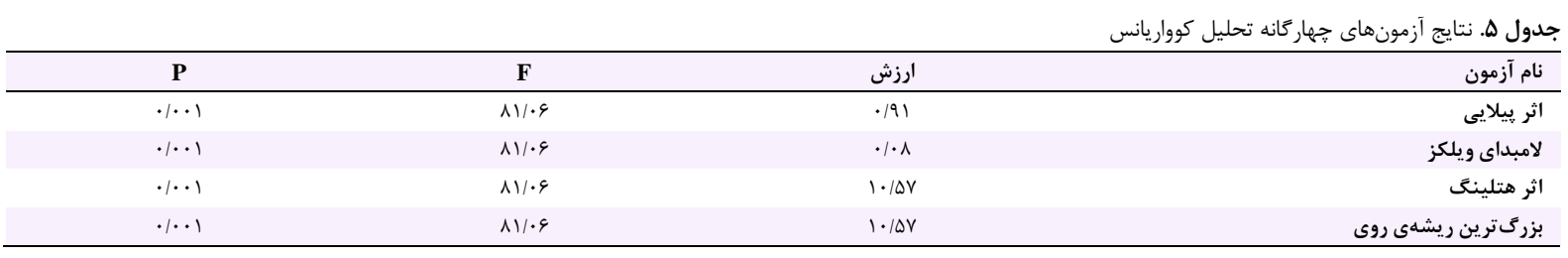

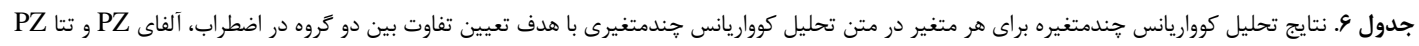

\begin{tabular}{|c|c|c|c|c|c|c|c|}
\hline توان آزمون & اندازه اثر & $\mathbf{P}$ & $\mathbf{F}$ & ميانكين مجذورات & درجه آزادى & مجموع مجذورات & منبع يراكندكى \\
\hline & & & & & & & يِيش آزمون \\
\hline$\cdot / 4 V$ & $\cdot 111$ & $\cdot 1 . .1$ & $r \cdot I / V F$ & qrV/DV & 1 & $q \mu V / \Delta V$ & اضطراب \\
\hline$\cdot / 1 T$ & $\cdot / \cdot V$ & $\cdot / \cdot 1$ & IVV/Ir & $r F I /$. & 1 & $r f I / l$. & آلفا PZ \\
\hline \multirow[t]{2}{*}{$\cdot / 19$} &.$/ 1 f$ &.$/ .1$ & TIV/DV & TrF/IF & 1 & rTF/lf & تتا PZ \\
\hline & & & & & & & تروه \\
\hline$\cdot \pi r$ & . & $\cdot 1 . .1$ & $199 / 99$ & $r \Delta 9 / 9 \Lambda$ & 1 & $r \Delta q / 9 \Lambda$ & اضطراب \\
\hline$\cdot / \pi 1$ & .148 & $\cdot 1 \cdot .1$ & $199 / \pi \Delta$ & $99 / 14$ & 1 & 99/14 & آلفا PZ \\
\hline \multirow[t]{5}{*}{$\cdot / K F$} & $\cdot / \pi 9$ & $\cdot 1 . .1$ & $\Delta \mathrm{N} / \cdot \mathrm{V}$ & FN/GT & 1 & FN/GY & Pت PZ PZ \\
\hline & & & & & & & خطا \\
\hline & & & & $\Delta r G / r G$ & Tr & DrG/TG & اضطراب \\
\hline & & & & NFD/AT & rV & $\Lambda F \Delta / \Lambda T$ & آلفا PZ \\
\hline & & & & rबr/9q & TV & TAT/9q & PZ تتا PZ \\
\hline
\end{tabular}

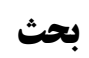

نتايج يزوهش حاضر نشان داد كه توانبخشى شناختى ميزان اضطراب اضراب

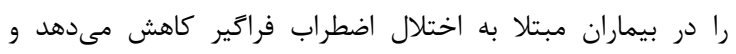

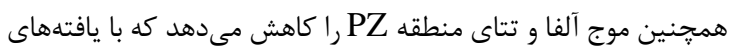

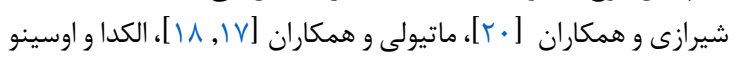

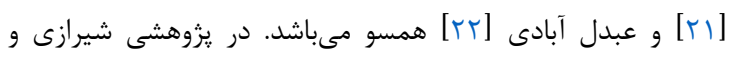

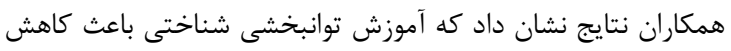

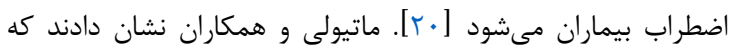

همان طور كه در جدول ب ملاحظه مىشود سطوح معنى دارى همه آماند

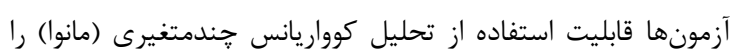

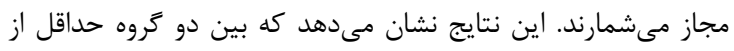

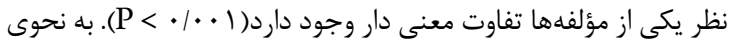

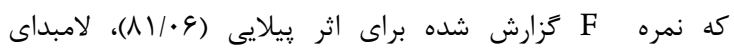

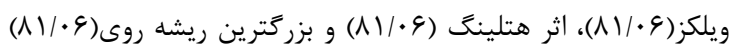

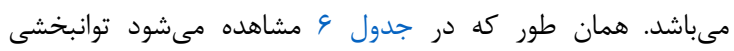

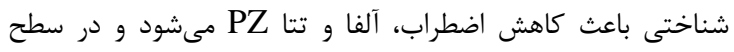

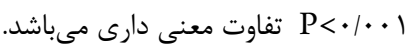


مىشود اثربخشى روش آموزش توانبخشى شناختى بر بهبود ساير

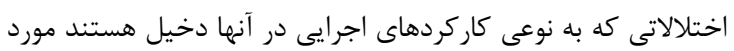

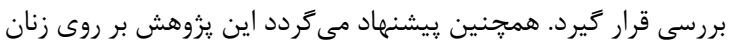

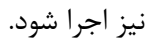

\section{نتيجه تيرى}

نتايج يزوهش حاضر نشان داد كه توانبخشى شناختى ميزان اضطراب

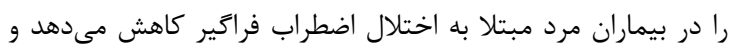

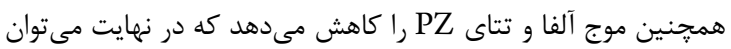

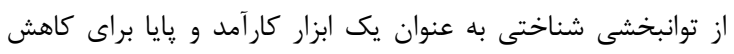

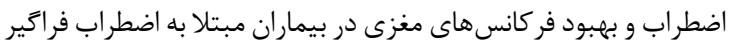

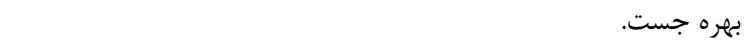
توانبخشى شناختى بر طبق اصل شكل يذيرى و خودترميمى مغزى، با فيا

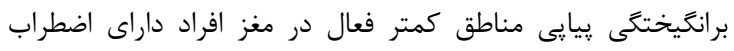

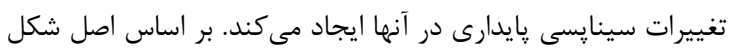

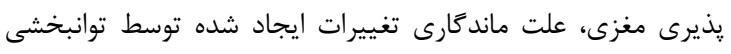

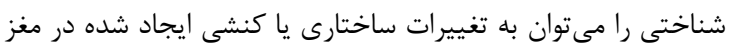

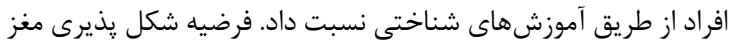

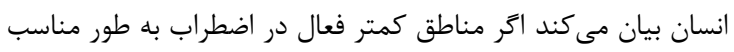

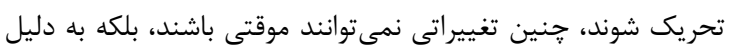
تغييراتى كه فرض مىشود در ساختار نورونها ايجاد كردهاند بايدار

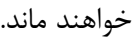

\section{ملاحظات اخلاقى}

در اين يزوهش از همه آزمودنىها رضايت نامه اخلاقى آكاهانه كسب آنات

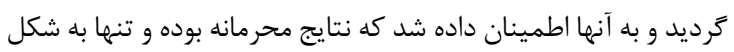
كروهى تحليل خواهند شد.

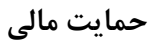

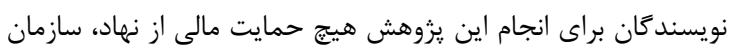

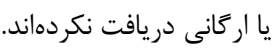

سهمم نويسندكان آقاى راهب جعفرى كار جمع آورى دادهها و نعارش مقاله را بر عهده

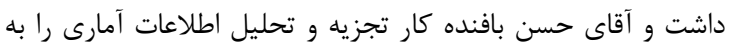

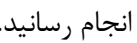

تعارض منافع

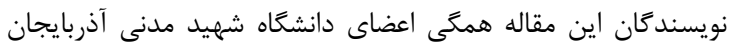

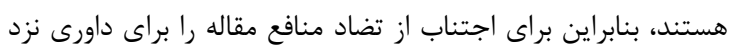
داوران عضو هيئت علمى دانشخاه مذكور ارسال نفرماييد.

كاربرد عملى مطالعه كاربرد علمى نتايج اين مطالعه مى تواند براى بيماران مبتلا به اضه اضطراب

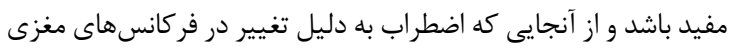

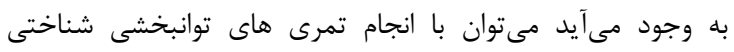

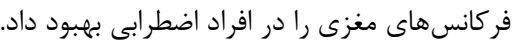

سياسگزارى

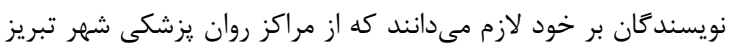

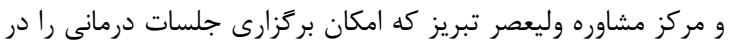

آموزش برنامه توانبخشى شناختى، امواج مغزى آلفا و تتا را تغيير مى دمى

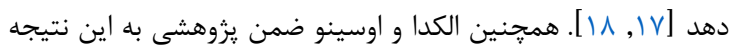

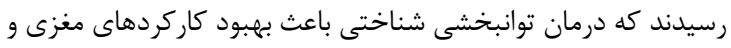

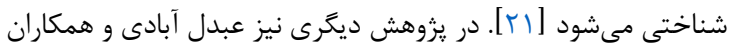

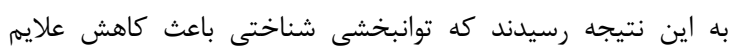

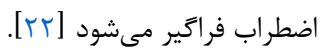

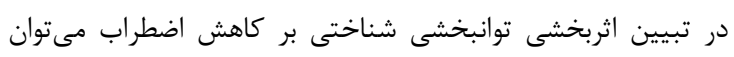

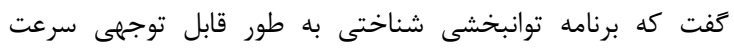

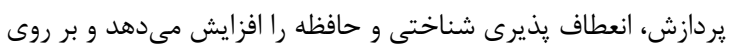

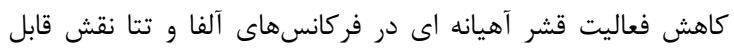

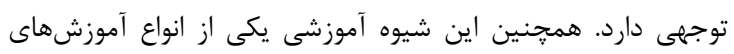

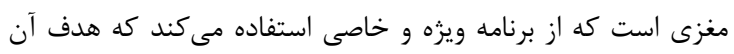

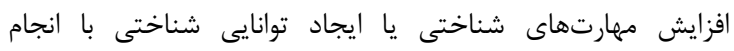

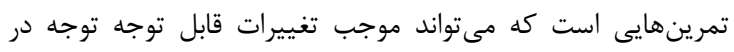

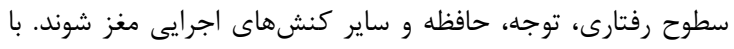

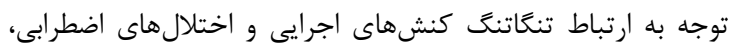

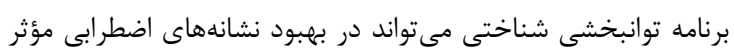

باشد [IV]

همجنين در تبيين اثربخشى توانبخشى بر كاهش فعاليت آلفا و تتاى

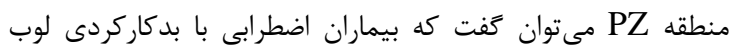

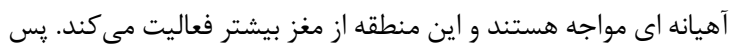

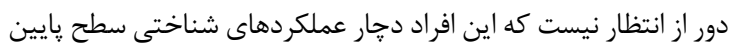

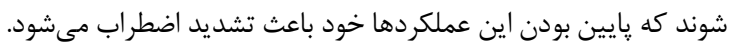

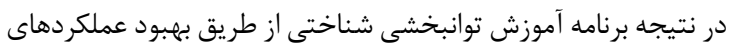

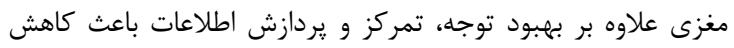

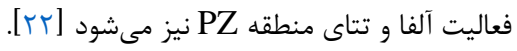

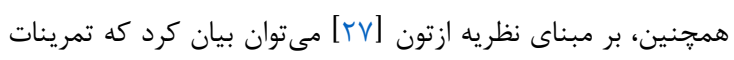

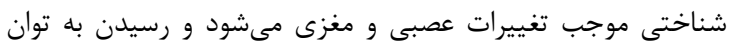

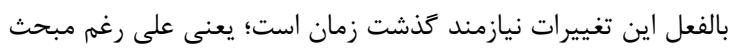

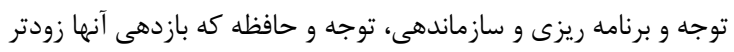

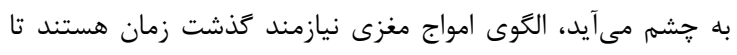

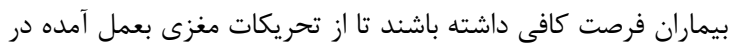

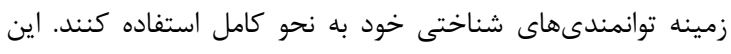

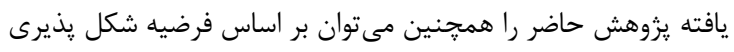

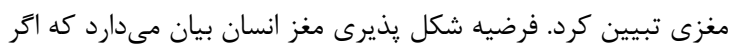

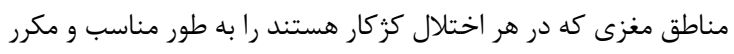

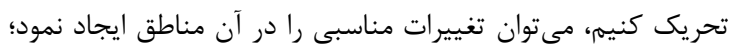

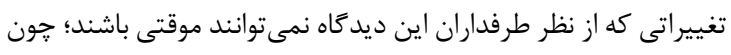

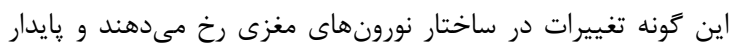

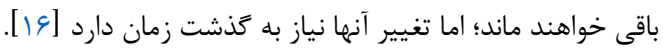

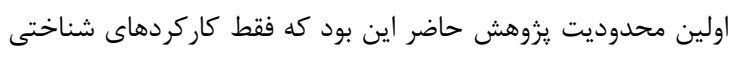

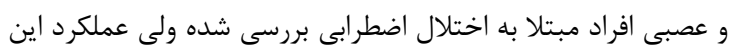

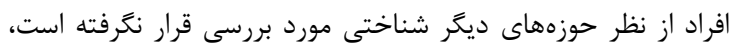

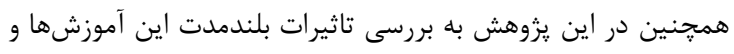

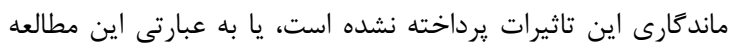

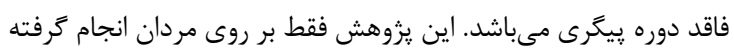

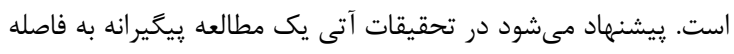

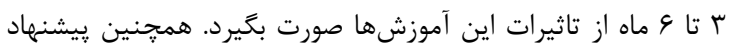




$$
\text { تشكر و قدردانى بعمل آوروندران قرار دادند و كليه شركت كنندكان در يُوهش حاضر }
$$

\section{References}

1. Arbab B, Vaez Mousavi SMK, Badami R. The relation between sports perfectionism and competitive anxiety in women's national karate team. [Persian]. J Res Sports Manage Motor Behav. 2016;5(10):27-35.

2. Hashim HA, Baghepour T. Validating the factorial structure of the Malaysian version of revised competitive state anxiety inventory-2 among young taekwondo athletes. Pertan J Soc Sci Hum. 2016;24(2):757-65.

3. Mehrsafar AH, Khabiri M, Moghaddamzadeh A. Factorial validity and reliability of Persian version of competitive state anxiety inventory-2 (CSAI-2) in intensity, direction and frequency dimensions. [Persian]. J Dev Motor Learn. 2016;8(2):253-79.

4. Roomruangwong C, Kanchanatawan B, Sirivichayakul S, Maes M. Antenatal depression and hematocrit levels as predictors of postpartum depression and anxiety symptoms. Psychiatry Res. 2016;238:211-7. doi: 10.1016/j.psychres.2016.02.039 pmid: 27086235

5. Niles AN, Dour HJ, Stanton AL, Roy-Byrne PP, Stein MB, Sullivan G, et al. Anxiety and depressive symptoms and medical illness among adults with anxiety disorders. J Psychosom Res. 2015;78(2):109-15. doi: 10.1016/j.jpsychores.2014.11.018 pmid: 25510186

6. McDonnell GA, Salley CG, Barnett M, DeRosa AP, Werk RS, Hourani A, et al. Anxiety Among Adolescent Survivors of Pediatric Cancer. J Adolesc Health. 2017;61(4):40923. doi: 10.1016/j.jadohealth.2017.04.004 pmid: 28729145

7. Ivan MC, Amspoker AB, Nadorff MR, Kunik ME, Cully JA, Wilson N, et al. Alcohol use, anxiety, and insomnia in older adults with generalized anxiety disorder. Am J Geriatr Psychiatry. 2014;22(9):875-83. doi: 10.1016/j.jagp.2013.04.001 pmid: 23973253

8. Driediger MV, McKay CD, Hall CR, Echlin PS. A qualitative examination of women's self-presentation and social physique anxiety during injury rehabilitation. Physiotherapy. 2016;102(4):371-6. doi: 10.1016/j.physio.2015.10.001 pmid: 26608591

9. Rahmanian M, Mohtarami S, Dehestani M. [The effect of cognitive rehabilitation training on improving anxiety symptoms in children]. J Child Ment. 2018;5(1):45-58.

10. Porcel J, Montalban X. Anticholinesterasics in the treatment of cognitive impairment in multiple sclerosis. J Neurol Sci. 2006;245(1-2):177-81. doi: 10.1016/j.jns.2005.07.021 pmid: 16674980

11. Arnett PA, Higginson CI, Randolph JJ. Depression in multiple sclerosis: relationship to planning ability. J Int Neuropsychol Soc. 2001;7(6):665-74. doi: 10.1017/s1355617701766027 pmid: 11575588

12. Moscati A, Flint J, Kendler KS. Classification of Anxiety Disorders Comorbid with Major Depression: Common or Distinct Influences on Risk? Depress Anxiety.
2016;33(2):120-7. doi: 10.1002/da.22432 pmid: 26418316

13. Canton-Cortes D, Cortes MR, Canton J. Child sexual abuse, attachment style, and depression: the role of the characteristics of abuse. J Interpers Violence. 2015;30(3):420-36. doi: 10.1177/0886260514535101 pmid: 24958133

14. Linares L, Jauregui P, Herrero-Fernandez D, Estevez A. Mediationg Role of Mindfulness as a Trait Between Attachment Styles and Depressive Symptoms. J Psychol. 2016;150(7):881-96. doi: 10.1080/00223980.2016.1207591 pmid: 27467235

15. Sahraian AM, Etesam F. Cognitive impairment in Multiple Sclerosis. [Persian]. Zahedan Univ Med Sci. 2014;16(1):1-7.

16. Cattelani R, Zettin M, Zoccolotti P. Rehabilitation treatments for adults with behavioral and psychosocial disorders following acquired brain injury: a systematic review. Neuropsychol Rev. 2010;20(1):52-85. doi: 10.1007/s11065-009-9125-y pmid: 20143264

17. Mattioli F, Stampatori C, Zanotti D, Parrinello G, Capra R. Efficacy and speci-ficity of intensive cognitive rehabilitation of attention and executive functionss in Multiple Sclerosis. J Neurol Sci. 2010;288(1-2):1015.

18. Mattioli F, Stampatori C, Scarpazza C, Parrinello G, Capra R. Persistence of the effects of attention and executive functions intensive rehabilitation in relapsing remitting multiple sclerosis. Mult Scler Relat Disord. 2012;1(4):168-73. doi: 10.1016/j.msard.2012.06.004 pmid: 25877265

19. Khalili L, Dovlatshahi B, Farhodi M, Niknam Z, Poorshabaz A. The effectiveness of attention rehabilitation in decreasing focused attention and [Persian]. J Urmia Univ Med Sci. 2014;25(5):382-96.

20. Shirazi M, Koohkan Azim H, Khosravani E. [Effectiveness of psychological rehabilitation, using Dohsa-Hou, on hemodialysis patients' depression, anxiety, and stress in Zahdan city]. Birjand Univ Med Sci. 2016;23(2):130-40.

21. Olukolade O, Osinowo HO. Efficacy of Cognitive Rehabilitation Therapy on Poststroke Depression among Survivors of First Stroke Attack in Ibadan, Nigeria. Behav Neurol. 2017;2017:4058124. doi: 10.1155/2017/4058124 pmid: 28720980

22. Bashi Abdolabadi H, Pilevar S, Saram AA. The Effect of Cognitive Rehabilitation on Cognitive Function, Memory, Depression, and Anxiety in Patients with Multiple Sclerosis. [Persian]. Neurosci J Shefaye Khatam. 2016;4(3):28-40. doi: 10.18869/acadpub.shefa.4.3.28

23. Beck AT, Steer RA, Carbin MG. Psychometric properties of the Beck Depression Inventory: Twenty-five years of working memory deficits on Multiple Sclerosis patients. 
evaluation. Clinic Psychol Rev. 1988;8(1):77-100. doi: 10.1016/0272-7358(88)90050-5

24. Mohammad Khani A, Bazpipour A. The relationship between activation and rejection systems of behavior with pathological and non-pathological worries. [Persian]. J Babol Univ Med Sci. 2010;12(1):59-64.

25. Hanasabzadeh Isfahani M, Yazdan-Doust F, Ghaarei B, Asgharnejad Farid A. Preliminary Study on the Effectiveness of Knowledge Based Cognitive Therapy Guide for Depressed Patients with Suicidal Thoughts: A Case Report. [Persian]. Clinic Psychol. 2009; 1(1):35-45.
26. Abbasi M, Ayadi N, Pirani Z, Montazeri Z. The Effect of Cognitive Rehabilitation on Social Well-Being, Quality of Life and Marital Satisfaction of Nurses. [Persian]. Iran J Health Educ Health Promot. 2017;5(3):231-9. doi: 10.30699/acadpub.ijhehp.5.3.231

27. Avzton S. Effect of neuroscience based cognitive skill training on growth of cognitive deficits associated with learning disabilities in children grades. Minneapolis: College of education, Walden University; 2012. 